\title{
Flux and resident injection in gaseous advection experiments
}

\author{
T. Gimmi ${ }^{1}$ and H. Flühler \\ Soil Physics, Institute of Terrestrial Ecology, Swiss Federal Institute of Technology, Zürich, Switzerland
}

\begin{abstract}
The occurrence of gaseous pollutants in soils has stimulated many experimental activities, including forced ventilation in the field as well as laboratory transport experiments with gases. The dispersion coefficient in advective-dispersive gas phase transport is often dominated by molecular diffusion, which leads to a large overall dispersivity $\gamma$. Under such conditions it is important to distinguish between flux and resident modes of solute injection and detection. The influence of the inlet type on the macroscopic injection mode was tested in two series of column experiments with gases at different mean flow velocities $v$. First we compared infinite resident and flux injections, and second, semi-infinite resident and flux injections. It is shown that the macroscopically apparent injection condition depends on the geometry of the inlet section. A reduction of the cross-sectional area of the inlet relative to that of the column is very effective in excluding the diffusive solute input, thus allowing us to use the solutions for a flux injection also at rather low mean flow velocities $v$. If the whole cross section of a column is exposed to a large reservoir like that of ambient air, a semi-infinite resident injection is established, which can be distinguished from a flux injection even at relatively high velocities $v$, depending on the mechanical dispersivity of the porous medium.
\end{abstract}

\section{Introduction}

The longitudinal dispersion coefficient $D_{L}$ in advectivedispersive gas phase transport is, in contrast to liquid phase transport, often dominated by molecular diffusion because of the relatively high gas phase diffusion coefficients. This is especially pronounced at low advection velocities when the mechanical dispersion, that is, the mixing due to structural properties, is small. This fact has several consequences regarding the input of solutes and the measurement of solute fluxes. In the region dominated by gaseous diffusion, the dispersivity $D_{L} / v$ is usually not constant. It is inversely proportional to the mean linear fluid velocity $v$ [Edwards and Richardson, 1968; Langer et al., 1978] and reaches therefore rather high values at relatively low advective velocities even in systems where the mechanical dispersivity is low. The differences between flux and resident concentrations as well as between flux and resident injections [Levenspiel and Turner, 1970; Kreft and Zuber, 1978; Parker and van Genuchten, 1984] are directly related to the value of the dispersivity and can therefore become rather large in advective-dispersive gas phase transport.

On the other hand, the large component of diffusive flux disturbs the injection and sampling of solutes proportional to the local fluid velocities, which is necessary for a correct flux injection or detection. For gaseous advective-dispersive transport, the exchange of solutes at a boundary will resemble a resident injection, unless the diffusive flux is restricted by geometrical constraints at the boundary. This creates a considerable uncertainty about the correct choice of model boundary conditions for specific laboratory or field experiments with gaseous tracers. The objective of this paper is to test the in-

\footnotetext{
${ }^{1}$ Now at Department of Soil and Environmental Sciences, University of California, Riverside.

Copyright 1996 by the American Geophysical Union.

Paper number 95WR02719.

0043-1397/96/95WR-02719\$05.00
}

fluence of the inlet type, and especially the inlet diameter, on the macroscopically apparent injection mode in gaseous advection experiments. Resident concentrations as a function of time are measured at different velocities for different inlets and compared with solutions for flux and resident injection.

\section{Summary of Injection Types}

A flux injection is characterized by imposing the solute flux at the entrance boundary, that is, at $x=0$. Based on mass balance considerations, this type of injection is usually considered to take place in column experiments [van Genuchten and Parker, 1984], often without being specified explicitly. In this case the flux concentration at the origin, $C_{f}\left(0^{+}, t\right)$, but not necessarily the resident concentration, $C_{r}\left(0^{+}, t\right)$, equals the concentration of the feeding solution, $C_{0}$ [Kreft and Zuber, 1978; Parker and van Genuchten, 1984]. We define the origin of the column at $x=0$, with the inlet located at negative and the column at positive $x$ values. The notation $x=0^{+}$indicates a location just at the beginning, but within the column. For an infinite, continuous resident injection, an infinite column extending from $x=-\infty$ to $+\infty$ is considered, where the resident concentration is imposed initially in the region $-\infty<x<0$ [Kreft and Zuber, 1978]. A semi-infinite, continuous resident injection into a column extending from $x=0$ to positive values is represented by a constant resident concentration at the origin. This semi-infinite resident injection is the actual counterpart of the flux injection. In the limiting case $v \rightarrow 0$, it is the correct solution for pure diffusion with a constant boundary concentration, whereas at this limit the solution for a flux injection represents a situation where no solutes enter the system. The boundary conditions for flux and resident concentrations for a flux, an infinite resident, or a semi-infinite resident injection and the corresponding solutions of the advective-dispersive equation (ADE) are summarized in Table 1. The origin of (5) and (6) for a semi-infinite resident injection is described in the appendix. (See Table 1 for (1)-(6).) 
Table 1. Boundary Conditions and Analytical Solutions of the One-Dimensional ADE for the Different Injection and Detection Types

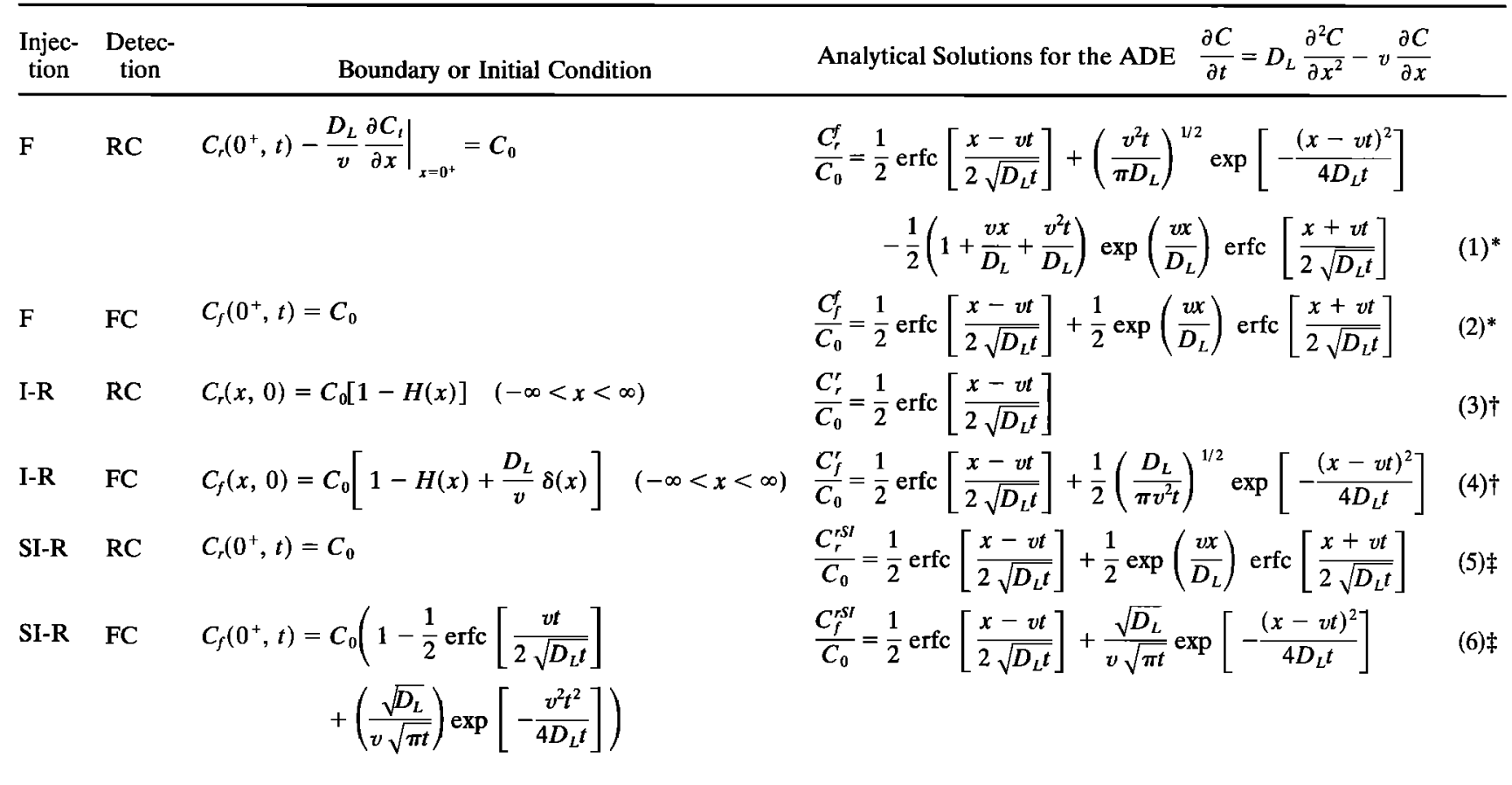

$\mathrm{ADE}$, advective-dispersive equation; $\mathrm{F}$, flux injection; I-R, infinite resident injection; SI-R, semi-infinite resident injection; FC, flux concentration; $\mathrm{RC}$, resident concentration. The lower boundary condition is in all cases $\partial C / \partial x(\infty, t)=0$; the initial condition, if not specified, is $C(x, 0)=0$.

${ }^{*}$ Kreft and Zuber [1978] and van Genuchten and Parker [1984].

$\dagger$ Kreft and Zuber [1978].

¥See appendix.

\section{Materials and Methods}

In most experiments we used a 0.5 -m-long plexiglass column with a diameter of $6 \mathrm{~cm}$. It was filled with solid glass beads, which had a density of $2.69 \mathrm{Mg} \mathrm{m}^{-3}$ and a mean radius of $1.20 \times 10^{-3} \mathrm{~m}$. The porosity of the medium ranged between 0.349 and 0.356 for the different experiments. Gaseous oxygen was used as a tracer and measured with oxygen electrodes usually at distances of 0.1 and $0.4 \mathrm{~m}$ from the inlet. It is believed that the response of oxygen electrodes placed at different locations in a column and facing the gaseous phase represents resident concentrations. The response is proportional to the local partial pressure of oxygen and is independent of the flow velocity of the air stream.

The experiments were performed at different, constant mean advective flow rates $v$, which were measured with a soap film flow meter. The typical response time of the electrodes sets an upper limit for the flow velocity, beyond which correct measurements are no longer possible. In our system, we had to keep the velocity below $10^{-2} \mathrm{~m} \mathrm{~s}^{-1}$, since otherwise the breakthrough occurred within a too short time to be measured accurately.

\section{Experimental Series I}

In a first series (Figure 1, I), the mechanical dispersivity $\alpha$ as well as the apparent diffusion coefficient $D_{m}$ were estimated from independent experiments (measurement distances 0.1 and $0.4 \mathrm{~m}$ ). The mechanical dispersivity was determined using a small (4-mm inner diameter (i.d.)) dead end tubing as inlet, which protruded slightly into the column and had four open- ings of $1 \mathrm{~mm}$ diameter on its side (Figure 1, top). The flow velocities were high enough to let all differences between the different injection types disappear $(P>25)$. The apparent diffusion coefficient was obtained from six experiments without advective flow, where the column was initially saturated with nitrogen and then exposed to the atmosphere on one side. The mechanical dispersivity $\alpha$ and the diffusion coefficient $D_{m}$ were fitted from these experiments by a nonlinear least squares procedure, where the objective function was minimized with the Levenberg-Marquardt algorithm [Press et al., 1992]. For the following experiments the dispersion coefficient $D_{L}$ was calculated from the velocity $v$, the mechanical dispersivity $\alpha$, and the apparent diffusion coefficient $D_{m}$ according to

$$
D_{L}=\alpha v+D_{m}
$$

\section{Experimental Series II}

In the second series of experiments (Figure 1, II) the column described above was attached to a precolumn, which was either a 0.25 -m-long column of the same diameter filled with glass beads (IIa) or a 1.5-m-long empty tubing with an i.d. of $12 \mathrm{~mm}$ (IIb), or a 1.5-m-long empty tubing with an i.d. of $6 \mathrm{~mm}$ (IIc). The experiment was started by joining center to center the 0.5-m-long column containing air with one of the three conduits previously saturated with nitrogen. Only a nylon mesh (mesh width of $1 \mathrm{~mm}$ ) with negligible flow resistance separated the precolumn or tubing and the $0.5-\mathrm{m}$-long main column. The joint columns were then flushed with nitrogen at various flow rates. The exit of the column consisted in all cases of a $2-\mathrm{m}-$ 

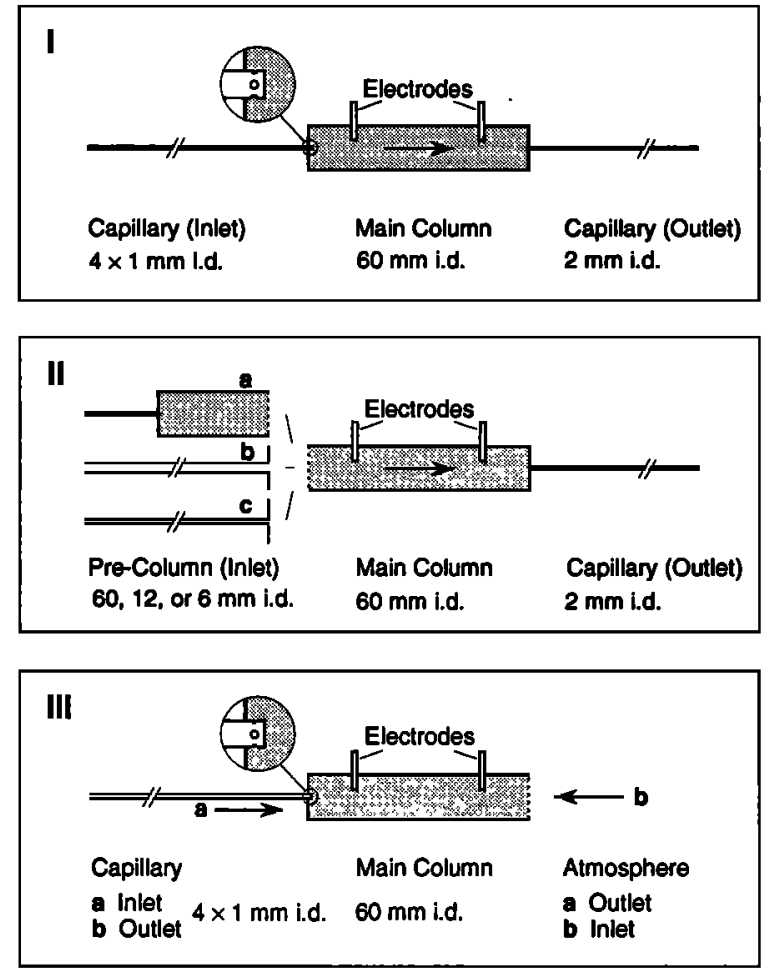

Figure 1. Schematic outline of the different series of advective-dispersive column experiments with gases. Roman numerals refer to experimental series. The dispersivity of the porous medium was determined using a small dead end tubing as inlet, which had four openings, each of 1-mm diameter, on its side (I). In the second series the inlet consisted of either a precolumn (IIa) or empty tubing (IIb and IIc). For series IIIa the same small tubing as in I was used as inlet, whereas for series IIIb the flow direction was reversed, such that the whole column cross section exposed to the ambient air served as inlet. The arrows indicate the flow direction, which, in the third series, was different for parts "a" and "b".

long small tubing (2-mm i.d.), which prevented backward diffusion and thus simulated a semi-infinite system.

\section{Experimental Series III}

For the third series of experiments (Figure 1, III), the 0.5-m column was connected again with the small tubing of 4-mm i.d. and four inlet openings of 1-mm diameter, whereas the other end was covered with a nylon mesh and left open to the atmosphere. Experiments were performed at three different, constant flow rates. At each flow rate an experiment consisted of two consecutive parts. First, nitrogen was flushed through the small tubing into the column initially containing ambient air (IIIa). The decrease of the oxygen concentration was monitored with the electrodes at a distance of 0.1 and $0.4 \mathrm{~m}$ from the inlet. Subsequently, the column was completely saturated with nitrogen by the aid of an exit valve. To start the second part of the experiment (IIIb), the valve was removed and the flow direction of the pump reversed. Thus the whole cross section of the column was used as inlet and exposed to ambient air. At that point the increase of the oxygen concentration was measured. This setup allowed us to keep all parameters constant and to test the influence of the boundary conditions only.

\section{Parameter Estimation}

The necessary parameters $D_{L}$ and $v$ were estimated independently (compare series I) for series II and for the low flow rate of series III. For the high flow rates of series III the velocity could not be measured reliably with the soap film flow meter. In this case $v$ as well as $D_{L}$ were fitted to measured data at $x=0.4 \mathrm{~m}$ for the first part (IIIa) of the experiment. The fitted parameter values were used afterwards to predict the second part (IIIb) of the experiment with the reversed flow direction and the whole cross section of the inlet being exposed to air.

\section{Modeling}

The experimental data were compared with the solutions for the various injection and detection types presented in Table 1. These solutions all represent semi-infinite systems. In the third series of experiments, the flow occurs first (IIIa) from the small tubing toward the open end, which represents a constant resident concentration at a finite distance. Therefore the ADE had to be solved for a finite system with a third-type inlet and a first-type outlet boundary condition, that is, for $C_{r}$ after a flux injection into a column of finite length with a constant resident concentration at the outlet end. The solution is obtained in the Laplace domain as

$$
\begin{aligned}
\hat{C}_{r}^{f}(x ; s)= & \frac{\exp [a(x-L)]}{s\left[\zeta_{2} \exp (b L)-\zeta_{1} \exp (-b L)\right]} \\
& \cdot\left\{C_{L}\left[\zeta_{2} \exp (b L)-\zeta_{1} \exp (-b L)\right]\right. \\
& \left.+2 C_{0} \exp (a L) \sinh [b(x-L)]\right\}
\end{aligned}
$$

where $a=v /\left(2 D_{L}\right), b=b(s)=a \xi(s), \xi(s)=\left(1+4 D_{L} s / v^{2}\right)^{1 / 2}, \zeta_{1,2}$ $=1 / 2 \pm(1 / 2) \xi(s), s$ is the Laplace variable, $C_{0}$ the input concentration, and $C_{L}$ the constant resident concentration at $x$ $=L$. The solution in the time domain is obtained by numerical inversion with the Talbot routine [Talbot, 1979; Jury and Roth, 1990], which is well suited for low Péclet numbers.

\section{Results and Discussion \\ Experimental Series I}

The apparent diffusion coefficient, $D_{m}=D_{m}^{o} / \tau$, of oxygen for the media with solid glass beads having a porosity of 0.349 to 0.356 was determined as $1.492 \times 10^{-5} \pm 3.9 \times 10^{-7} \mathrm{~m}^{2} \mathrm{~s}^{-1}$ $\left(T=22^{\circ} \mathrm{C}\right.$ ). The binary diffusion coefficient $D_{m}^{o}$ for oxygen in nitrogen at this temperature is $2.074 \times 10^{-5} \mathrm{~m}^{2} \mathrm{~s}^{-1}$ [Marrero and Mason, 1972]; thus the tortuosity $\tau$ of the column packing is calculated as 1.39 . The mechanical gas dispersivity as determined from several runs at flow rates $v$ around $5 \times 10^{-3} \mathrm{~m} \mathrm{~s}^{-1}$ $(P \approx 100)$ was $\alpha=1.06 \times 10^{-3} \mathrm{~m}$. This agrees well with the dispersivity value estimated as the mean radius of the beads $\left(1.20 \times 10^{-3} \mathrm{~m}\right)$ after Edwards and Richardson [1968]. It was, however, not possible to use velocities high enough to be in the range of a clearly dominant dynamic dispersion (i.e., $\alpha / \beta \gg 1$, where $\beta=D_{m} / v$ is the molecular dispersivity, or $v>10^{-2} \mathrm{~m}$ $\mathrm{s}^{-1}$ for this medium) because of the limited response time of the electrodes, as can be seen from Figure 2. Thus the uncertainty of the experimental $\alpha$ value, estimated from the uncertainties of $D_{m}$ and of the fitted $D_{L}$ and $v$, is quite large (standard deviation of $\sim 4.5 \times 10^{-4} \mathrm{~m}$ ). In all experiments the dispersion coefficient was largely determined by molecular diffusion, and thus $P=v l / D_{L} \approx v l / D_{m}$, where $l$ is the measurement distance from the inlet end. 


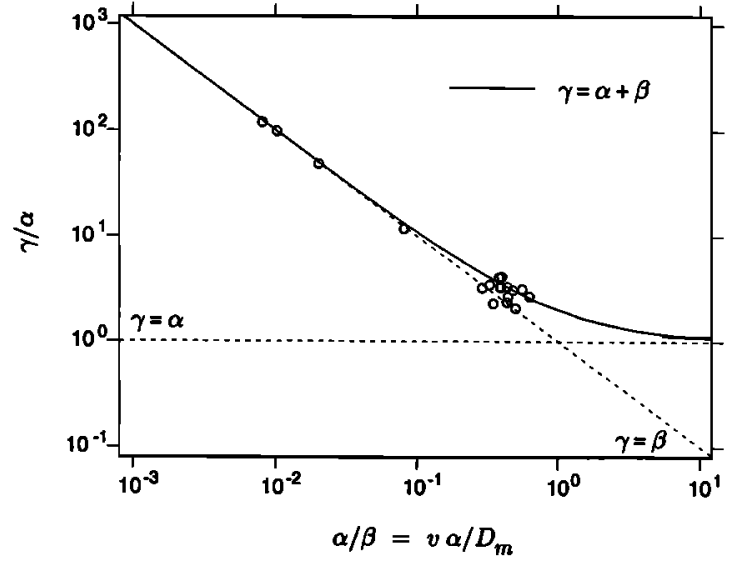

Figure 2. Dispersivity values $\gamma=D_{L} / v$ of all experiments, scaled with the mechanical dispersivity $\alpha$, as a function of $\alpha / \beta=$ $v \alpha / D_{m}$. The values $\gamma / \alpha<10$ were used to estimate the mechanical dispersivity $\alpha$. The solid line assumes additivity of the mechanical $(\alpha)$ and the molecular $(\beta)$ diffusivities. It can be seen that diffusion dominated the overall dispersion in all experiments.

\section{Experimental Series II}

Figures 3 to 6 show results of the second series of experiments (IIa-IIc). The precolumn (IIa), initially saturated with nitrogen, and the main column, both packed with glass beads, were flushed at a relatively high velocity of $v=4.90 \times 10^{-5} \mathrm{~m}$ $\mathrm{s}^{-1}$ (Figure 3, $P=0.33$ and 1.3). At least initially, this leads to an infinite resident injection according to (3). The ratio between the cross sections of the precolumn or inlet, $A_{i}$, and of the column, $A_{c}$, is in this case unity. A reduction of the inlet diameter from 60 to $12 \mathrm{~mm}\left(A_{i} / A_{c}=0.04\right.$, IIb) leads to a different picture. For a similar velocity of $v=3.91 \times 10^{-5} \mathrm{~m}$ $\mathrm{s}^{-1}(P=0.26$ and 1.0$)$ a flux injection according to (1) seems to be achieved (Figure 4), whereas a lower mean linear velocity of $v=3.19 \times 10^{-6} \mathrm{~m} \mathrm{~s}^{-1}(P=0.021$ and 0.086$)$ leads to a concentration increase, which is characteristic for a resident

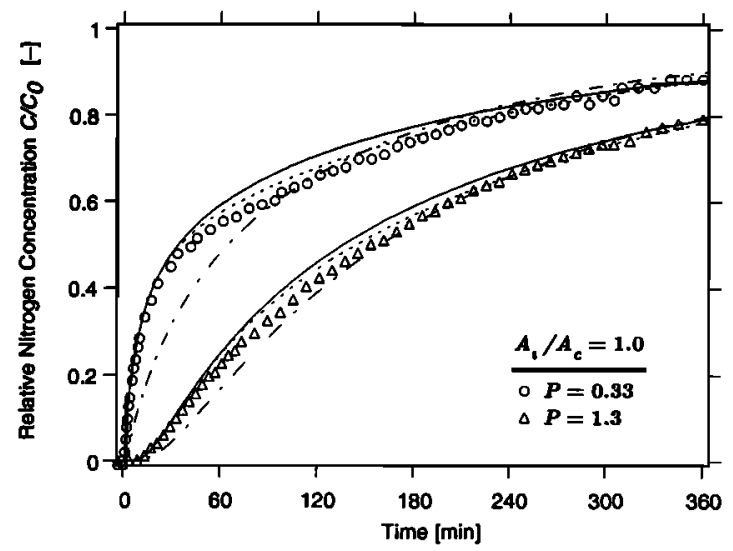

Figure 3. Breakthrough of nitrogen measured at $x=0.1$ (circles) and $0.4 \mathrm{~m}$ (triangles) for $\nu=4.9 \times 10^{-5} \mathrm{~m} \mathrm{~s}^{-1}$. A precolumn of the same diameter (experimental series IIa, $A_{\imath} / A_{c}=1$ ), initially saturated with nitrogen, is used as inlet. Calculations represent an infinite resident injection (solid line), a flux injection (dashed dotted line), and a special case (see text) accounting for the finite length of the precolumn (dashed line).

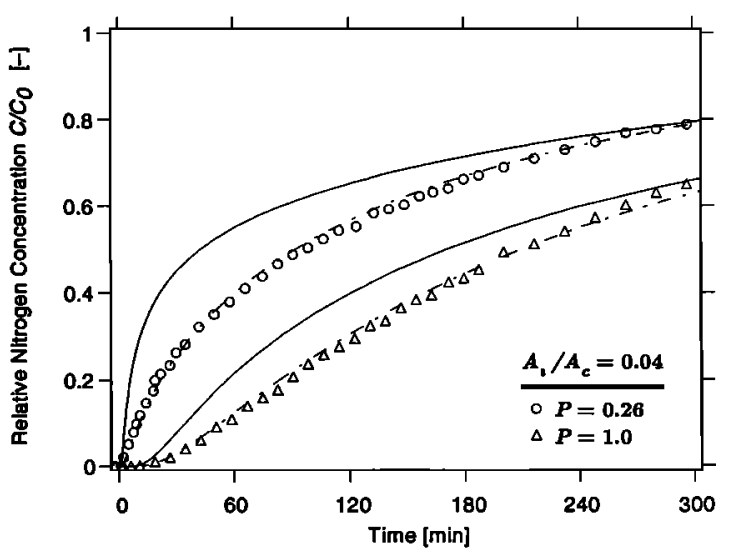

Figure 4. Breakthrough of nitrogen measured at $x=0.1$ (circles) and $0.4 \mathrm{~m}$ (triangles), $v=3.9 \times 10^{-5} \mathrm{~m} \mathrm{~s}^{-1}$. The inlet has a diameter of $12 \mathrm{~mm}$ (IIb, $A_{i} / A_{c}=0.04$ ). Calculations represent an infinite resident (solid line) and a flux injection (dashed line).

injection (Figure 5). The measured increase does not of course exactly follow the calculations for an infinite resident injection, because the column and the inlet have different cross sections. Using an even smaller inlet diameter of $6 \mathrm{~mm}\left(A_{t} / A_{c}=0.01\right.$, IIc) leads to a flux injection also at a flow rate of $v=3.74 \times 10^{-6}$ $\mathrm{m} \mathrm{s}^{-1}(P=0.025$ and 0.10$)$, as it can be seen in Figure 6 .

This second series of experiments shows that macroscopically, a flux injection can be imposed also at rather low flow rates by using a small tubing as inlet conduit. The reduction of the cross section of the inlet compared to the cross section of the column is very efficient. The diffusive flux at the boundary is clearly restricted, and its effect may appear, if at all, only at very early times.

The experiment with the precolumn filled with glass beads (IIa, Figure 3) represents a special case. The differences between measurements and curves calculated for an infinite resident injection (solid lines) after about $30 \mathrm{~min}$ at $x=0.1 \mathrm{~m}$ originate from the finite length of the precolumn initially saturated with nitrogen. After a certain time the injection type at the entrance of the precolumn becomes dominant. There the

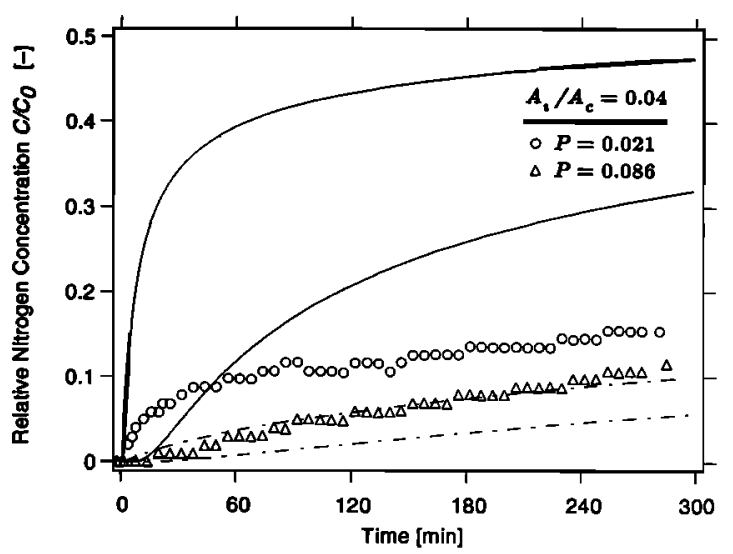

Figure 5. Breakthrough of nitrogen measured at $x=0.1$ (circles) and $0.4 \mathrm{~m}$ (triangles) for $v=3.2 \times 10^{-6} \mathrm{~m} \mathrm{~s}^{-1}$. The inlet has a diameter of $12 \mathrm{~mm}\left(\mathrm{IIb}, A_{c} / A_{c}=0.04\right)$. Calculations represent an infinite resident (solid line) and a flux injection (dashed line). 


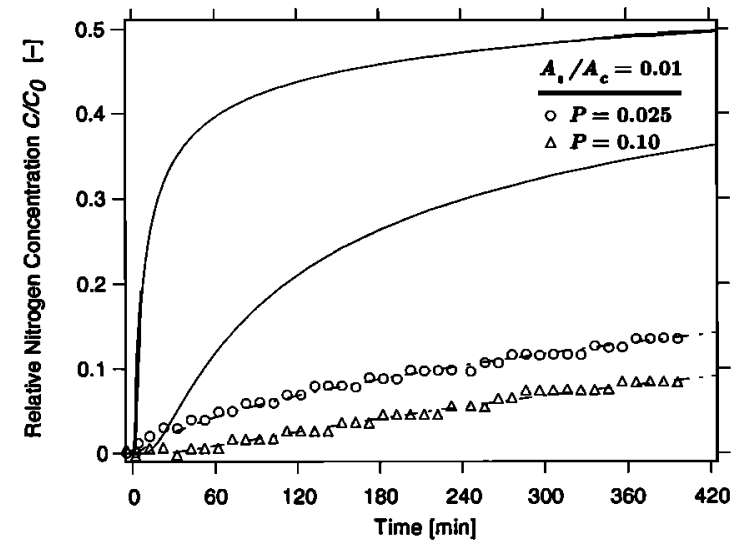

Figure 6. Breakthrough of nitrogen measured at $x=0.1$ (circles) and $0.4 \mathrm{~m}$ (triangles) for $v=3.7 \times 10^{-6} \mathrm{~m} \mathrm{~s}^{-1}$. The inlet has a diameter of $6 \mathrm{~mm}$ (IIc, $A_{\mathrm{z}} / A_{c}=0.01$ ). Calculations represent an infinite resident (solid line) and a flux injection (dashed line).

fluid is injected through a small tubing, which leads to a flux injection at this velocity. This can be verified by comparing the measurements with a solution for the combined columns, accounting for an upper boundary condition of the third type and an initial step distribution in a semi-infinite column. This solution [van Genuchten and Alves, 1982, solution (A6)], which is shown as dashed lines in Figure 3 , follows the data also at larger times.

It is obvious that the ratio $A_{l} / A_{c}$, as well as the dispersivity of the porous medium and possibly the differences in dispersivities between column and precolumn, determine which solution is appropriate and which type of injection macroscopically occurs. For a flux injection we have to require that the influence of diffusion is negligible compared to mechanical dispersion. This means that $\beta f<\alpha$, where $\alpha$ is the mechanical and $\beta$ the molecular dispersivity. The factor $f$ depends on the geometry of the inlet section, which introduces usually additional resistances to diffusive mixing compared to dispersive. The diffusive mass exchange at the interface is limited to the cross sectional area of the inlet, $A_{\iota}$, whereas the dispersive term is proportional to $\alpha v A_{c}$, which is constant for a given column unless density changes occur. Therefore as a first approximation we can estimate $f$ as $A_{\imath} / A_{c}$. Expressing the condition $\beta f<\alpha$ for $v$, we obtain

$$
v>\frac{f D_{m}^{o}}{\alpha \tau}
$$

If $v$ exceeds this limit, the purely dispersive flux component at the inlet is always larger than the diffusive, leading to a proper flux injection. According to this relation and for the given mechanical dispersivity and diffusion coefficient in our experiments, the minimum value of $v$ that is required for a correct flux injection is approximately $1 \times 10^{-2}, 5 \times 10^{-4}$, and $1 \times$ $10^{-4} \mathrm{~m} \mathrm{~s}^{-1}$ for $A_{\imath} / A_{c}$ of $1,0.04$, and 0.01 , respectively. The experiments did show that for $A_{t} / A_{c}<1$, a flux injection is achieved already at lower velocities of $v=3.91 \times 10^{-5} \mathrm{~m} \mathrm{~s}^{-1}$ $\left(A_{t} / A_{c}=0.04\right)$ and $v=3.74 \times 10^{-6} \mathrm{~m} \mathrm{~s}^{-1}\left(A_{i} / A_{c}=0.01\right)$, which means that $f<A_{t} / A_{c}$. This can be explained by the fact that at larger times the advective component of the flux at the inlet dominates for all injection types at all velocities, as illustrated in Figure 7 for a flux injection. In this figure the diffusive

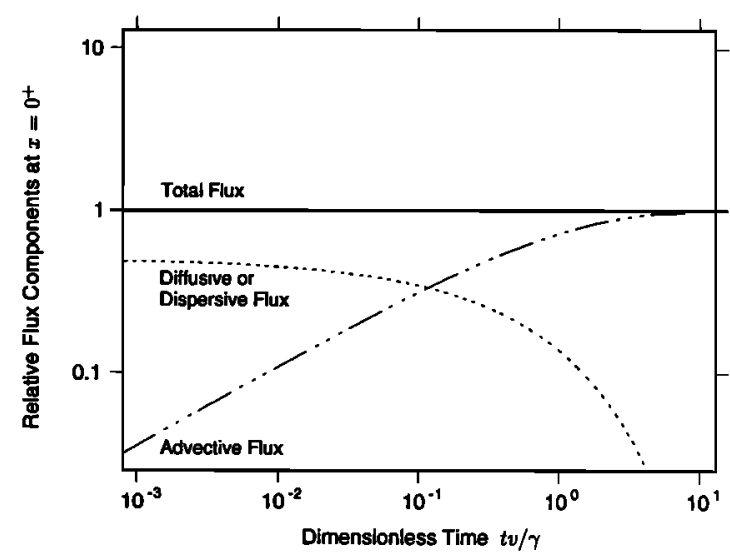

Figure 7. Magnitudes of flux components at the inlet relative to the total flux versus dimensionless time $t v / \gamma$ for a flux injection. The diffusive flux component is set equal to the flux caused by mechanical dispersion $(\alpha=f \beta)$. Total (solid line), diffusive or dispersive (dashed line), and advective flux components (dashed dotted line) are shown.

and the advective flux components at the inlet are compared with the total flux, the diffusive and dispersive fluxes being equally important (i.e., $\alpha=f \beta$ ). The corresponding plots for a resident injection look similar. For larger $v$ the time $t \approx$ $0.1 \gamma / v$ until advection dominates becomes rather short, and the diffusive solute input during this experimental period is of minor importance. By using $f=A_{i} / A_{c}$ in (9), one is certainly on the safe side if a flux injection in a given experimental system is intended.

\section{Experimental Series III}

The results of the third series of experiments, carried out at three different velocities, are depicted in Figures 8 to 10. To allow direct comparisons, the breakthroughs of nitrogen (labeled "a") and oxygen (labeled "b") are shown. For each

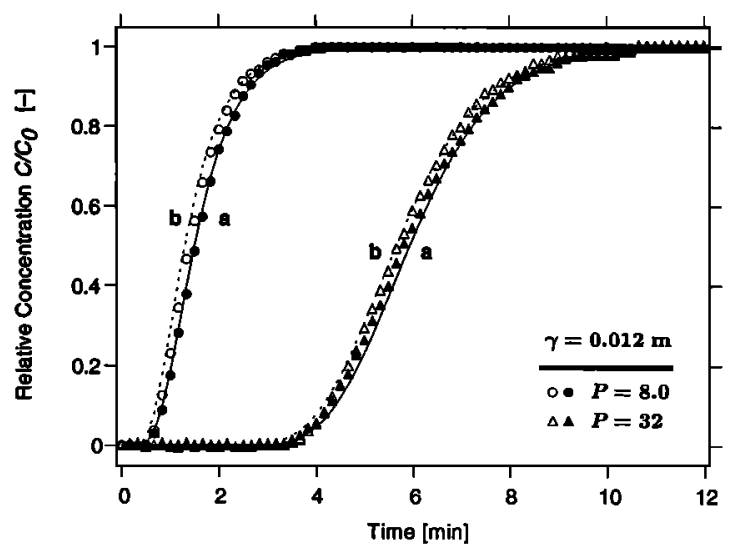

Figure 8. Influence of the physical boundary conditions on the breakthrough behavior at a relatively high velocity $\left(v=1.14 \times 10^{-3} \mathrm{~m} \mathrm{~s}^{-1}, \gamma=0.012 \mathrm{~m}\right)$, measured at $x=0.1$ m (circles; $P=8.0$ ) and $x=0.4 \mathrm{~m}$ (triangles; $P=32$ ). The solution (8) for a flux injection, $C_{r}^{f}$ (solid line), is fitted to the experimental data of part IIIa (closed symbols; small inlet) at $x=0.4 \mathrm{~m}$. The fitted parameters are used to predict part IIIb of the experiment (open symbols; whole cross section of column exposed to air) with solution (5) for a semi-infinite resident injection, $C_{r}^{r S I}$ (dashed line). 


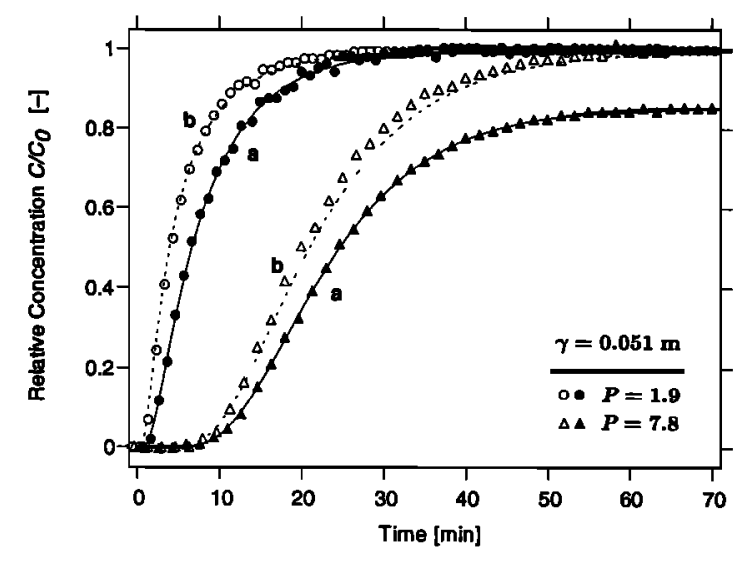

Figure 9. Influence of the experimental boundary conditions on the breakthrough behavior at a medium velocity ( $v=2.82$ $\times 10^{-4} \mathrm{~m} \mathrm{~s}^{-1}, \gamma=0.051 \mathrm{~m}$ ), measured at $x=0.1 \mathrm{~m}$ (circles; $P=1.9$ ) and $x=0.4 \mathrm{~m}$ (triangles; $P=7.8$ ). The solution (8) for a flux injection into a finite column, $C_{r}^{f}$ (solid line), is fitted to the experimental data of part IIIa (closed symbols; small inlet) at $x=0.4 \mathrm{~m}$. The fitted parameters are used to predict part IIIb of the experiment (open symbols; whole cross section of column exposed to air) with solution (5) for a semiinfinite resident injection, $C_{r}^{r S}$ (dashed line).

velocity the breakthrough of nitrogen is measured at a distance of 0.1 and $0.4 \mathrm{~m}$ using the small tubing as inlet (IIIa, solid symbols). Assuming that the experiment can be described by a flux injection and in terms of resident concentrations, that is by $C_{r}^{f}$ for a finite system (8), a good fit between model calculations and breakthrough curves was obtained for the higher velocities of $v=1.14 \times 10^{-3} \mathrm{~m} \mathrm{~s}^{-1}$ (Figure $8, \gamma=0.012 \mathrm{~m}$ ) and $v=$ $2.82 \times 10^{-4} \mathrm{~m} \mathrm{~s}^{-1}$ (Figure 9, $\gamma=0.051 \mathrm{~m}$ ). The second part of the experiment (IIIb, open symbols), with a flow of oxygen from the open end, could be well predicted with the fitted

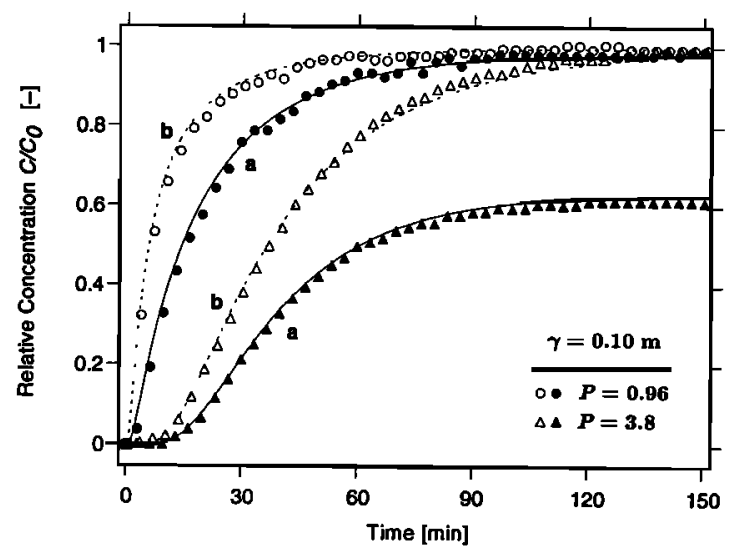

Figure 10. Influence of the experimental boundary conditions on the breakthrough behavior at a relatively low velocity $\left(v=1.44 \times 10^{-4} \mathrm{~m} \mathrm{~s}^{-1}, \gamma=0.10 \mathrm{~m}\right)$, measured at $x=0.1 \mathrm{~m}$ (circles; $P=0.96$ ) and $x=0.4 \mathrm{~m}$ (triangles; $P=3.8$ ). The experimental values of part IIIa (closed symbols; small inlet) are compared to solution (8) for a flux injection into a finite column, $C_{r}^{f}$ (solid line), whereas part IIIb (open symbols; whole cross section of column exposed to air) is compared to solution (5) for a semi-infinite resident injection, $C_{r}^{r S r}$ (dashed line). The model parameters are determined independently for both flow directions, IIIa and IIIb. parameters, using the solution for $C_{r}^{r S I}(5)$, that is a resident concentration originating from a continuous, semi-infinite resident injection (Figures 8 and 9). For the lowest velocity $\left(v=1.44 \times 10^{-4} \mathrm{~m} \mathrm{~s}^{-1}, \gamma=0.10 \mathrm{~m}\right)$ the data of both flow directions (IIIa and IIIb) could be well predicted with the independently estimated parameters and the corresponding solutions for a flux or a semi-infinite, continuous resident injection (Figure 10).

For the largest velocity the differences between the two solutions as well as between the two measured breakthrough curves (IIIa) and (IIIb) are already rather small because of the relatively high Péclet numbers $(P=8.0$ and 32$)$. For the lower velocities $(P=1.9$ and 7.8 , and $P=0.96$ and 3.8$)$ the differences are substantial even if we ignore the effects caused by the finite size of the column in part (IIIa) of the experiments. The effects of the finite size of the column manifest at $x=0.4 \mathrm{~m}$ by a steady state concentration $\left.C_{r}^{f}\right|_{t \rightarrow \infty}<C_{0}$. It must be emphasized that the solutions for the two parts of the experiment are obtained with an upper boundary condition of the third (IIIa) or the first type (IIIb), as it is usually done to calculate resident or flux concentrations in column experiments. Here, in both cases the same measurement device is used, which obviously determines resident concentrations. The differences are therefore only caused by the injection types, that is, by a flux (IIIa) versus a semi-infinite resident injection with a constant concentration (IIIb).

The case where the whole cross section of the column is used as an inlet and exposed to a large volume of ambient air represents a situation with a cross sections ratio, $A_{\imath} / A_{c}$, larger than 1 . Then the condition to achieve with certainty a flux injection, $v>\left(A_{\imath} / A_{c}\right)\left(D_{m}^{o} / \alpha \tau\right)$, can only be met at very high velocities. This leads to high Péclet numbers, at which the different solutions coincide anyway. Experimentally, a semiinfinite resident injection with a constant resident concentration at the boundary was observed and could be distinguished from a flux injection as long as measurable differences in the mathematical solutions did exist.

\section{Summary and Conclusions}

The possibly diffusive exchange of solutes across boundaries in gaseous, advective-diffusive flow requires a critical analysis of the appropriate mathematical boundary conditions, which must be specified in terms of flux or resident mode [Kreft and Zuber, 1978; van Genuchten and Parker, 1984]. Column experiments with gases showed that the macroscopically prevalent injection conditions depend on the geometry of the inlet section. The factor $f$, which accounts for effects of the geometry, is correlated with the ratio of the inlet cross section to the column cross section, $A_{\imath} / A_{c}$. For a constricted inlet it seems that $f<A_{\imath} / A_{c}$, whereas for an open inlet $f \geq 1$. If the condition $v>\left(A_{\imath} / A_{c}\right)\left(D_{m}^{o} / \alpha \tau\right)$ is met, for instance by using a very thin conduit as an inlet, a flux injection is achieved with certainty, and (1) and (2) can be used to analyze the experimental data. If, on the other hand, $v$ is considerably lower than $\left(A_{t} / A_{c}\right)\left(D_{m}^{o} / \alpha \tau\right)$, the solute input is initially larger than expected for a flux injection, and the concentrations within the column increase faster. In this case it will no longer be accurate to estimate parameters like the dispersivity from tracer experiments, using the solution for a flux injection. Doing so will result in erroneous parameter values.

A continuous semi-infinite resident injection, which is characterized by a constant resident concentration at the origin, 
was experimentally observed when the whole cross section of the column was exposed to the ambient air. Although neither backward diffusion influenced the concentration $C_{0}$, and there did not exist a concentration gradient at $x<0$ (well-stirred reservoir, $\left.C(x<0, t)=C_{0}\right)$, a resident, not a flux, injection developed at all velocities that allowed a distinction of the different solutions. This situation corresponds exactly with the exchange of solutes between the atmosphere and the soil gas phase. In general, the larger mechanical dispersivity $\alpha$ of real soil compared with the glass beads will render mechanical dispersion slightly more important but not yet dominating. Only at very high gaseous flow rates, like, for instance in soil venting, a flux injection might really occur at the soil surface. But at such high flow rates the differences between the injection types become negligible, anyway. In most cases involving lower gas flow rates, it will presumably be possible to model the gas exchange as a semi-infinite resident injection with a continuous resident concentration at the soil surface, unless the mixing within the atmosphere is strongly restricted by vegetation.

\section{Appendix}

A relatively large diffusive solute flux at the inlet boundary at $x=0$ can lead to macroscopically continuous resident concentrations

$$
C\left(0^{+}, t\right)=C_{0}
$$

The solution of the advective-dispersive equation (ADE) for this boundary condition in a semi-infinite column, (5) in Table 1 , is formally identical with (2) of Table 1 , but with $C_{f}^{f}$ replaced by $C_{r}^{r S I}$, that is, the resident concentration in case of a continuous semi-infinite resident injection. This solution is also known as the Lapidus and Amundson [1952] or Ogata and Banks [1961] solution. The corresponding equation for the flux concentration, $C_{f}^{r S I}$, can be obtained through the relation

$$
C_{f}=C_{r}-\frac{D_{L}}{v} \frac{\partial C_{r}}{\partial x}
$$

where $D_{L} / v$ is the overall dispersivity. This relation between the two detection modes (flux concentration or weighted solute flux on one hand, and resident concentration on the other hand) is valid for the advective-dispersive transport model if both $C_{f}$ and $C_{r}$ relate to the same injection type. To obtain $C_{f}^{r S I}$, we can plug the solution (5) for $C_{r}^{r S I}$ as well as the gradient $\partial C_{r}^{r S I} / \partial x$ calculated from (5) directly into (A2). This leads to the solution presented in (6) (Table 1) and the corresponding boundary condition.

\section{Notation}

The subscripts $f$ and $r$ denote detection in flux or in resident fluid, respectively. The superscripts $f, r$, and $r S I$ stand for a flux injection, a resident injection, or a semi-infinite resident injection. For instance, $C_{r}^{f}$ is a resident concentration obtained in case of a flux injection.

$$
\begin{array}{ll}
A_{\iota} & \text { cross-sectional area of inlet }\left[L^{2}\right] . \\
A_{c} & \text { cross-sectional area of column }\left[L^{2}\right] .
\end{array}
$$

$C$ concentration $\left[M L^{-3}\right]$.

$C_{0}$ concentration of the feeding solution at $x=0^{-}$or initial resident concentration at $x<0\left[M L^{-3}\right]$.

$D_{L}$ longitudinal dispersion coefficient, including molecular diffusion and mechanical dispersion $\left[L^{2} T^{-1}\right]$.

$D_{m}$ effective molecular diffusion coefficient of porous medium, equal to $D_{m}^{o} / \tau\left[L^{2} T^{-1}\right]$.

$D_{m}^{o}$ binary molecular diffusion coefficient $\left[L^{2} T^{-1}\right]$.

$l$ reference or measurement distance $[L]$.

$P$ column Péclet number, equal to $v l / D_{L}=l / \gamma$ [dimensionless].

$t$ time [T].

$v$ average linear velocity of fluid phase $\left[L T^{-1}\right]$.

$x$ distance $[L]$.

\section{Greek Symbols}

$\alpha \quad$ mechanical dispersivity $[L]$.

$\beta$ molecular dispersivity, equal to $D_{m}^{o} /(\tau v)[L]$.

$\gamma$ overall dispersivity, equal to $D_{L} / v=\alpha+\beta[L]$.

$\tau$ fluid phase tortuosity [dimensionless].

\section{References}

Edwards, M. F., and J. F. Richardson, Gas dispersion in packed beds, Chem. Eng. Sci., 23, 109-123, 1968.

Jury, W. A., and K. Roth, Transfer Functions and Solute Movement Through Soil: Theory and Applications, Birkhäuser, Basel, 1990.

Kreft, A., and A. Zuber, On the physical meaning of the dispersion equation and its solutions for different initial and boundary conditions, Chem. Eng. Sci., 33, 1471-1480, 1978.

Langer, G., A. Roethe, K. P. Roethe, and D. Gelbin, Heat and mass transfer in packed beds, III, Axial mass dispersion, Int. J. Heat Mass Transfer, 21, 751-759, 1978.

Lapidus, L., and N. R. Amundson, Mathematics of adsorption in beds, VI, The effect of longitudinal dispersion in ion exchange and chromatographic columns, J. Phys. Chem., 56, 984-988, 1952.

Levenspiel, $O$., and J. C. R. Turner, The interpretation of residencetime experiments, Chem. Eng. Sci., 25, 1605-1609, 1970.

Marrero, T. R., and E. A. Mason, Gaseous diffusion coefficients, $J$. Phys. Chem. Ref. Data, 1, 3-118, 1972.

Ogata, A., and R. B. Banks, A solution of the differential equation of longitudinal dispersion in porous media, U.S. Geol. Surv. Prof. Pap., 411-A, A1-A9, 1961.

Parker, J. C., and M. T. van Genuchten, Flux-averaged and volumeaveraged concentrations in continuum approaches to solute transport, Water Resour. Res., 20, 866-872, 1984.

Press, W. H., S. A. Teukolsky, W. T. Vetterling, and B. P. Flannery, Numerical recipes: The Art of Scientific Computing, 2nd ed., Cambridge Univ. Press, New York, 1992.

Talbot, A., The accurate numerical inversion of Laplace transforms, $J$. Inst. Math. Its Appl., 23, 97-120, 1979.

van Genuchten, M. T., and W. J. Alves, Analytical solutions of the one-dimensional convective-dispersive solute transport equation, Tech. Bull. 1661, U.S. Dep. of Agric., Washington, D. C., 1982.

van Genuchten, M. T., and J. C. Parker, Boundary conditions for displacement experiments through short laboratory soil columns, Soil Sci. Soc. Am. J., 48, 703-708, 1984.

H. Flühler, Soil Physics, Institute of Terrestrial Ecology, Swiss Federal Institute of Technology, ETHZ, Grabenstrasse 3, CH-8952 Schlieren, Switzerland.

T. Gimmi, Department of Soil and Environmental Sciences, University of California, Riverside, CA 92521. (email: gimmi@dustside.ucr.edu)

(Received January 31, 1995; revised August 16, 1995; accepted August 31, 1995.) 